Network Working Group

Request for Comments: 3306

Category: Standards Track
B. Haberman Consultant

D. Thaler Microsoft

August 2002

\title{
Unicast-Prefix-based IPv6 Multicast Addresses
}

\section{Status of this Memo}

This document specifies an Internet standards track protocol for the Internet community, and requests discussion and suggestions for improvements. Please refer to the current edition of the "Internet Official Protocol Standards" (STD 1) for the standardization state and status of this protocol. Distribution of this memo is unlimited.

Copyright Notice

Copyright (C) The Internet Society (2002). All Rights Reserved.

Abstract

This specification defines an extension to the multicast addressing architecture of the IP Version 6 protocol. The extension presented in this document allows for unicast-prefix-based allocation of multicast addresses. By delegating multicast addresses at the same time as unicast prefixes, network operators will be able to identify their multicast addresses without needing to run an inter-domain allocation protocol.

Table of Contents

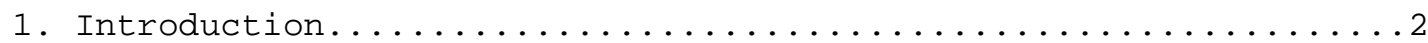

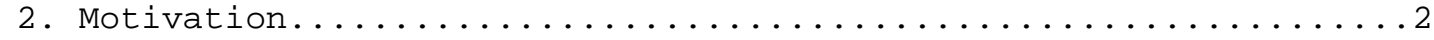

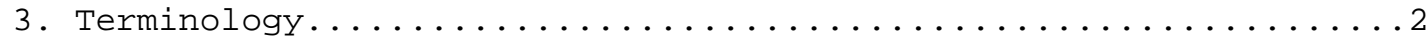

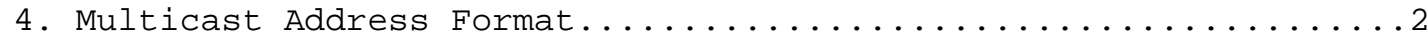

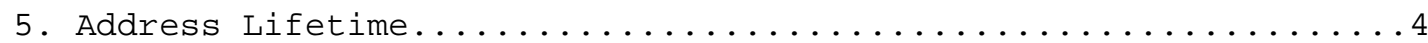

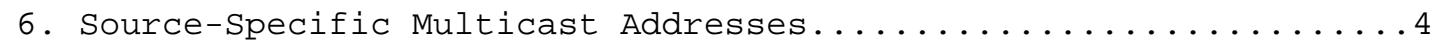

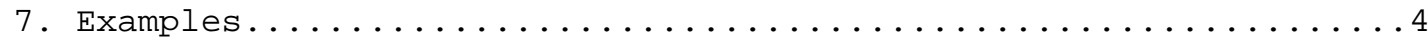

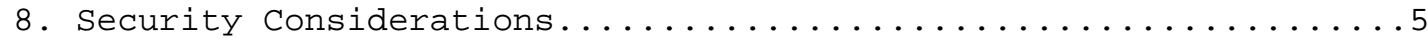

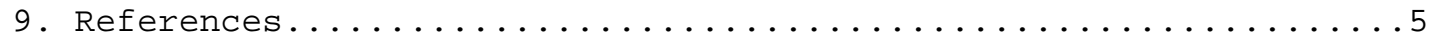

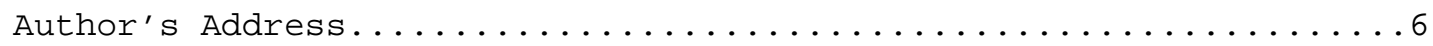

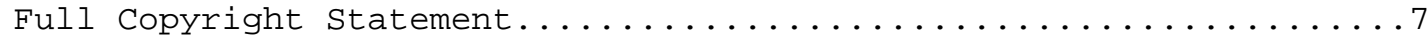


1. Introduction

This document specifies an extension to the multicast portion of the IPv6 addressing architecture [ADDRARCH]. The current architecture does not contain any built-in support for dynamic address allocation. This proposal introduces encoded information in the multicast address to allow for dynamic allocation of IPv6 multicast addresses and IPv6 source-specific multicast addresses.

2. Motivation

The current IPv4 multicast address allocation architecture [RFC 2908] is based on a multi-layered, multi-protocol system. The goal of this proposal is to reduce the number of protocols that need to be deployed in order to get dynamic multicast address allocation.

The use of unicast prefix-based multicast address allocation will, at a minimum, remove the need to run the Multicast Address Allocation Protocol (AAP) [AAP WORK] and the Multicast Address-Set Claim (MASC) Protocol [RFC 2909].

3. Terminology

The key words "MUST", "MUST NOT", "REQUIRED", "SHALL", "SHALL NOT", "SHOULD", "SHOULD NOT", "RECOMMENDED", "MAY", and "OPTIONAL" in this document are to be interpreted as described in [RFC 2119].

4. Multicast Address Format

Section 2.7 of [ADDRARCH] defines the following operational format of IPv6 multicast addresses:

\begin{tabular}{|c|c|c|}
\hline 8 & \begin{tabular}{l|l}
4 & 4
\end{tabular} & 112 \\
\hline 11111111 & |flgs $\mid$ scop $\mid$ & group ID \\
\hline
\end{tabular}


This document introduces a new format that incorporates unicast prefix information in the multicast address. The following illustrates the new format:

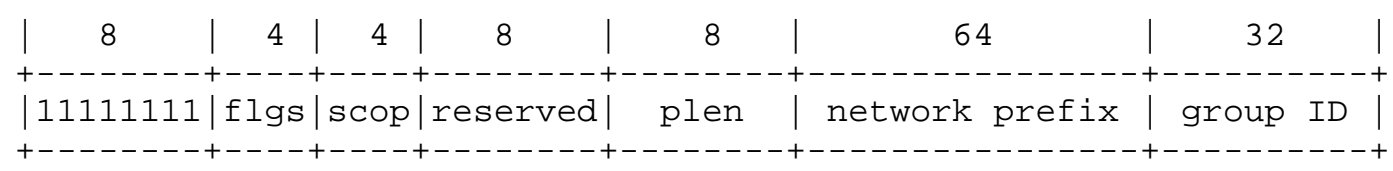

flgs is a set of 4 flags: $\quad \begin{aligned} & |0| 0|\mathrm{P}| \mathrm{T} \mid \\ & +-+-+-+-+\end{aligned}$

- $\mathrm{P}=0$ indicates a multicast address that is not assigned based on the network prefix. This indicates a multicast address as defined in [ADDRARCH].

- $\mathrm{P}=1$ indicates a multicast address that is assigned based on the network prefix.

o If $\mathrm{P}=1$, $\mathrm{T}$ MUST be set to 1 , otherwise the setting of the $\mathrm{T}$ bit is defined in Section 2.7 of [ADDRARCH].

The reserved field MUST be zero.

plen indicates the actual number of bits in the network prefix field that identify the subnet when $\mathrm{P}=1$.

network prefix identifies the network prefix of the unicast subnet owning the multicast address. If $P=1$, this field contains the unicast network prefix assigned to the domain owning, or allocating, the multicast address. All non-significant bits of the network prefix field SHOULD be zero.

It should be noted that the Interface Identifier requirements in Section 2.5.1 of [ADDRARCH] effectively restrict the length of the unicast prefix to 64 bits, hence the network prefix portion of the multicast address will be at most 64 bits.

Group ID is set based on the guidelines outlined in [IPV6 GID].

The scope of the unicast-prefix based multicast address MUST NOT exceed the scope of the unicast prefix embedded in the multicast address. 


\section{Address Lifetime}

The lifetime of a unicast prefix-based multicast address SHOULD NOT exceed the Valid Lifetime field in the Prefix Information option, corresponding to the unicast prefix being used, contained in the Neighbor Discovery Router Advertisement message [RFC 2461]. The lifetime of the multicast address is needed to support the Abstract API for Multicast Address Allocation [RFC 2771].

It should be noted that the unicast prefix's Valid Lifetime in the Router Advertisement message does not indicate that the prefix will become invalid at the end of the lifetime. Rather, that value is typically a constant until a renumbering event is scheduled after which, the prefix does become invalid.

The use of unicast prefix-based multicast addresses after the unicast prefix has become invalid may lead to operational problems. For example, routers that perform policy checks comparing the multicast prefix against the unicast prefix assigned to an AS may discard the packet.

6. Source-Specific Multicast Addresses

The unicast prefix-based IPV6 multicast address format supports Source-specific multicast addresses, as defined by [SSM ARCH]. To accomplish this, a node MUST:

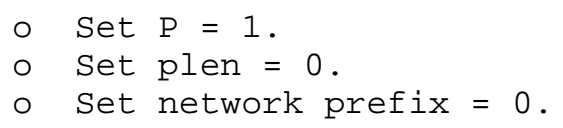

These settings create an SSM range of FF3x::/32 (where ' $x$ ' is any valid scope value). The source address field in the IPv6 header identifies the owner of the multicast address.

7. Examples

The following are a few examples of the structure of unicast prefixbased multicast addresses.

- Global prefixes - A network with a unicast prefix of 3FFE:FFFF:1::/48 would also have a unicast prefix-based multicast prefix of FF3x:0030:3FFE:FFFF:0001::/96 (where ' $x^{\prime}$ is any valid scope).

- SSM - All IPv6 SSM multicast addresses will have the format FF3x: : /96. 
8. Security Considerations

It is possible that the embedded unicast prefix can aid in identifying the allocation domain of a given multicast address, though an allocation domain choosing to avoid being traced has no obstacles currently to creating addresses using a prefix not assigned to it, or using a smaller scope embedded prefix.

Using source-specific multicast addresses can sometimes aid in the prevention of denial-of-service attacks by arbitrary sources, although no guarantee is provided. A more in-depth discussion of the security considerations for SSM can be found in [SSM ARCH] .

9. References

[RFC 2026] Bradner, S., "The Internet Standards Process -- Revision 3", BCP 9, RFC 2026, October 1996.

[RFC 2460] Deering, S. and R. Hinden, "Internet Protocol, Version 6 (IPv6) Specification", RFC 2460, December 1998.

[ADDRARCH] Hinden, R. and S. Deering, "IP Version 6 Addressing Architecture", RFC 2373, July 1998.

[RFC 2908] Thaler, D., Handley, M. and D. Estrin, "The Internet Multicast Address Allocation Architecture", RFC 2908, September 2000 .

[AAP WORK] Handley, M. and S. Hanna, "Multicast Address Allocation Protocol (AAP)", Work In Progress.

[RFC 2909] Radoslavov, P., Estrin, D., Govindan, R., Handley, M., Kumar, S. and D. Thaler, "The Multicast Address-Set Claim (MASC) Protocol", RFC 2909, September 2000.

[RFC 2119] Bradner, S., "Key words for use in RFCs to Indicate Requirement Levels", BCP 14, RFC 2119, March 1999.

[IPV6 GID] Haberman, B., "Dynamic Allocation Guidelines for IPv6 Multicast Addresses", RFC 3307, June 2002.

[RFC 2461] Narten, T., Nordmark, E. and W. Simpson, "Neighbor Discovery for IP Version 6 (IPv6)", RFC 2461, December 1998 .

[RFC 2771] Finlayson, R., "An Abstract API for Multicast Address Allocation", RFC 2771, February 2000. 
[SSM ARCH] Holbrook, H. and B. Cain, "Source-Specific Multicast for IP", Work In Progress.

Author's Address

Brian Haberman

Consultant

Phone: 1-919-949-4828

EMail: bkhabs@nc.rr.com

Dave Thaler

Microsoft Corporation

One Microsoft Way

Redmond, WA 48105-6399

Phone: 1-425-703-8835

EMail: dthaleremicrosoft.com 
Full Copyright statement

Copyright (C) The Internet Society (2002). All Rights Reserved.

This document and translations of it may be copied and furnished to others, and derivative works that comment on or otherwise explain it or assist in its implementation may be prepared, copied, published and distributed, in whole or in part, without restriction of any kind, provided that the above copyright notice and this paragraph are included on all such copies and derivative works. However, this document itself may not be modified in any way, such as by removing the copyright notice or references to the Internet society or other Internet organizations, except as needed for the purpose of developing Internet standards in which case the procedures for copyrights defined in the Internet Standards process must be followed, or as required to translate it into languages other than English.

The limited permissions granted above are perpetual and will not be revoked by the Internet society or its successors or assigns.

This document and the information contained herein is provided on an "AS IS" basis and THE INTERNET SOCIETY AND THE INTERNET ENGINEERING TASK FORCE DISCLAIMS ALL WARRANTIES, EXPRESS OR IMPLIED, INCLUDING BUT NOT LIMITED TO ANY WARRANTY THAT THE USE OF THE INFORMATION HEREIN WILL NOT INFRINGE ANY RIGHTS OR ANY IMPLIED WARRANTIES OF MERCHANTABILITY OR FITNESS FOR A PARTICULAR PURPOSE.

Acknowledgement

Funding for the RFC Editor function is currently provided by the Internet Society. 